\title{
INTEGRATION OF LUTI MODELS INTO SUSTAINABLE URBAN MOBILITY PLANS (SUMPS)
}

\author{
NIKOLAOS GAVANAS ${ }^{1}$, GEORGIA POZOUKIDOU ${ }^{2, *}$, \\ and ELENI VERANI ${ }^{1}$
}

\author{
${ }^{1}$ Transport Engineering Laboratory, School of Civil Engineering, Aristotle University of Thessaloniki, University Campus, \\ Faculty of Engineering, 54124 Thessaloniki, Greece \\ ${ }^{2}$ School of Spatial Planning and Development, Aristotle University of Thessaloniki, University campus, Faculty of Engineering, \\ 54124 Thessaloniki, Greece \\ *Corresponding author: gpozoukid@plandevel.auth.gr
}

\section{ABSTRACT}

\begin{abstract}
A literature review indicates that there is an increasing number of Land Use/Transport Interaction (LUTI) models being used in policy analysis and support of urban land use, transport and environmental planning. In this context, LUTI models are considered to be useful for the development of scenarios during the preparatory stage of Sustainable Urban Mobility Plans (SUMPs). A SUMP can be defined as a strategic planning framework, proposed by the European Commission, for planning and design of an urban multimodal transport system, which combines multi-disciplinary policy analysis and decision making. The objective of a SUMP is to achieve sustainable urban mobility, i.e. accessibility for all, safety and security, reduction in emissions and energy consumption, efficient and cost-effective transport and an improvement in the urban environment. Based on the overall conceptual and methodological framework of LUTI models (Geurs and van Wee 2004), the scope of the proposed research is to fully integrate a LUTI model into a contemporary transport planning framework and, more specifically, into the SUMP structure. This paper focuses on the configuration of the integration pattern, according to which a LUTI model may evolve and interact with the planning process throughout the eleven elements of the SUMP, as well as the evaluation of the benefits and drawbacks from the implementation of the proposed pattern for the enhancement of SUMP and overall promotion of sustainable urban planning.
\end{abstract}

Keywords: land use and transport integrated model, sustainable urban mobility plan, integration, interaction, evaluation

doi: $10.14712 / 23361964.2016 .3$

\section{Introduction}

The land use system illustrates the spatial organization of the network of socio-economic activities and describes the physical separation between them. The transportation system connects the various activities/land uses, but at the same time, leads to new mobility and accessibility conditions that may create new time-space relationship between land-uses (Rodrigue 2013). In addition, transport infrastructure consumes a significant part of the available space, especially in urban areas, and may produce fragmentation and segregation effects (Seiler and Folkenson 2006; EEA 2013).

The analysis of the interaction between the two systems: transport and land use is nowadays established as a core issue of mobility planning due to the emergence of the concept of sustainable mobility. In opposition to traditional urban transport planning, where the increasing demand in mobility coped with the constant increase in infrastructure, sustainable urban mobility planning is a more holistic approach that aims at the maximization of the efficiency of the transportation system and minimization of externalities, i.e., environmental degradation.

The investigation of the interaction between transport infrastructure and spatial development is based on two different methods (Pitsiava-Latinopoulou and Zaharaki 2004): (a) the empirical studies 'before' and 'after' the construction of a transport project and (b) the Land Use/ Transport Interaction (LUTI) models. A LUTI model is a tool for supporting strategic planning by estimating trends in locational choices and forecasting land use patterns by combining features such as mobility patterns, socio-demographic characteristics, industry allocation, geomorphological and environmental factors, availability of urban networks and institutional and policy frameworks (Pozoukidou 2010). Recently LUTI models have attracted the attention of the scientific community in terms of their role in strategic transport planning, since they are considered to be the most appropriate tool for achieving an understanding of the cause and effect relationship between transport and land use.

At the same time the European Commission (EC) promotes the aforementioned sustainable planning approach for urban mobility within the framework of Sustainable Urban Mobility Plans (SUMPs) (European Commission 2013). A SUMP is a strategic plan that fosters a balanced development of all modes of urban transport, while encouraging a shift towards more sustainable modes, by a combination of inter-disciplinary planning and policy analysis, and decision making. Its objectives coincide with the components of sustainable mobility, i.e., accessibility for all, efficient and affordable mobility services, enhancement of safety and security, decrease of emissions and improvement of energy efficiency and an improvement in the urban environment. More specifically, it covers the whole planning process from the preparatory and goal setting stages to the elaboration and implementation stages through a series of elements that correspond to the specific objectives of the plan, each comprising a set of activities. The plan unravels in 
a circular pattern and concludes by setting the basis for the implementation of the next SUMP (Bührmann et al. 2013).

The SUMP guidelines recommend a combination of appropriate techniques, such as quality management and benchmarking, with efficient tools, such as indicators and models, for the successful implementation of the activities and fulfilment of the objectives. For this it is suggested that the use of a LUTI model during the preparatory stage of the SUMP could provide an analysis of the effect of the transportation system on locational choices (Bührmann et al. 2013). Based on this suggestion, the objective of the current paper is to investigate the possibility of integrating a LUTI model into the SUMP cycle.

\section{Integration of a LUTI Model into a SUMP}

\section{A Brief Description of Sustainable Urban Mobility Plans}

The European Commission promoted the Sustainable Urban Mobility Plan (SUMP) as the common framework for the strategic planning for sustainable mobility in $\mathrm{Eu}$ ropean cities (European Commission 2013). In this context, the implementation of SUMP is promoted through policy documentation, programmes, initiatives and financial support instruments related to the objectives of sustainable transportation and urban development. The main feature of a SUMP is the integration of:

- The decision-making mechanism by embedding the main European strategies for sustainable mobility (European Commission 2009) and ensuring the involvement and commitment of national and local authorities, stakeholders and society through the allocation of responsibilities and public consultation.

- Successful inter-disciplinary planning approaches, such as the Plans de Deplacements Urbains implemented in France and the Local Transport Plans implemented in the United Kingdom.

- Planning and evaluation tools, such as monitoring indicators, forecasting models, SWOT (Strengths-Weaknesses-Opportunities-Threats) analysis, etc.

- Priorities and measures for all transport modes in terms of balanced competition and co-modality.

The goals of sustainable development are central to setting the priorities of a SUMP. In particular, a SUMP should be able to describe a long-term and comprehensive set of priorities and related measures for the: a) Social inclusion in the provision of efficient and affordable transport services, b) Improvement of the safety and security of transport activities, c) Enhancement of the quality of the urban and natural environment, the decrease of the transport system's carbon footprint and management of natural resources and energy and d) Economic development and strengthening of competitiveness.

A SUMP unravels in a circular way the whole process of strategic planning, i.e. the identification of the general scope, the formulation of specific goals and targets, the analysis and forecasting of mobility features, the definition and implementation of measures and the monitoring and evaluation of effects. In this way, the plan ends with an update and review of the implementation results and identification of the key-features that will lead to the implementation of another SUMP cycle. According to the guidelines (Bührmann et al. 2011), the aforementioned cycle includes the following 4 phases and 11 corresponding steps:

\section{A. Preparation}

1. Investigation of potentials

1.1 Commit to overall sustainable mobility plans

1.2 Assessment of effect of regional/national framework

1.3 Conduct self-assessment

1.4 Review of availability of resources

1.5 Definition of a basic timeline

1.6 Identification of key actors and stakeholders

2. Definition of the development process and scope of the plan

2.1 Look beyond your own boundaries and responsibilities

2.2 Strive for policy coordination and an integrated planning approach

2.3 Planning of stakeholder and citizen involvement

2.4 Agreement on a work plan and management arrangements

3. Analysis of the mobility conditions and development of scenarios

3.1 Preparation of an analysis of problems and opportunities

3.2 Development of scenarios

B. Goal setting

4. Development of a vision for sustainable urban mobility

4.1 Development of a common vision of mobility and beyond

4.2 Actively inform the public

5. Setting of priorities and measurable targets

5.1 Identification of the priorities for mobility

5.2 Development of smart targets

6. Definition of measures

6.1 Identification of the most effective measures

6.2 Learning from others' experience

6.3 Consideration of the best value for money

6.4 Use synergies and create integrated packages of measures

C. Elaboration

7. Allocation of responsibilities and resources

7.1 Assignment of responsibilities and resources

7.2 Preparation of an action and budget plan

8. Formulation of the monitoring and assessment process

8.1 Monitoring and evaluation process

9. Adoption of the SUMP

9.1 Check the quality of the plan

9.2 Adoption of the plan 
9.3 Create ownership of the plan (official adoption of the plan with the involvement of citizens and stakeholders)

\section{Implementation}

10. Management and communication

10.1 Management of the implementation of the plan

10.2 Information and engagement of citizens

10.3 Check progress towards achieving the objectives

11. Overall evaluation

11.1 Update the current plan regularly

11.2 Review the achievements - understand success and failure

11.3 Identification of new challenges for generating the next SUMP

Each step comprises a set of activities that are essential for achieving the milestones of the corresponding phase, i.e. the conclusion of the analysis of problems and opportunities, the identification of measures, the adoption of the plan and conclusion of the assessment of the final effect.

\section{Land Use Transport Interaction Models}

Land use/Transport interaction models (LUTI) are the way planners use the capabilities of personal computers to process quickly, reliably and accurately large volumes of spatial data. The first generation of LUTI models were developed in North America in 1950s, where rapid economic growth and the need for systematic study of the interactions between land use and the transport system set the conditions for the creation and exploitation of the first urban models (Brail and Klosterman 2001). Since then the use of LUTI models, is in many cases, a prerequisite to long-term and medium-term strategic plans for sustainable urban development. International literature refers in great detail to the use and utility of LUTI models, as tools for evaluating the effect of urban development policies (U.S. EPA 2000; Spiekermann and Wegener 2004). Actually it was the passage of two federal policies in USA in the early 1990s (Clean Air Act and Intermodal Surface Transportation Act) that introduced the LUTI models used by academics into the planning practice.

LUTI are spatial interaction models used in urban models of a planning process, the first generation of which were static synthetic economic and spatial interaction models. The theoretical background of these models is based on regional economics, locational theories and urban economics. Therefore they embody the principle of land suitability, as a result of the interaction between economic production factors. In particular, it was Von Thunen in 1826 that set the basis for locational theories by making the simple assumption that production of goods at a certain site will continue only if the profit from this activity (profit minus production cost) is greater than the cost of the transport of goods. Much later in 1964, Alonso incorporated in this theory the principle of land suitability and land bid rent curves for household and business, while Sinclair in 1967 used the theory of Von Thunen to explain the phenomenon of urban sprawl.

From a mathematical point of view choice of location is determined by exponential or logarithmic models based on the method of utility maximization and entropy or random utility by setting limitations to the cost of transport determined, in most cases, by income. These mathematical models calculate the probability of occurrence / establishment of a particular type of land use or urban function taking into account all of the factors mentioned above (transport, rent, etc.). They incorpo-

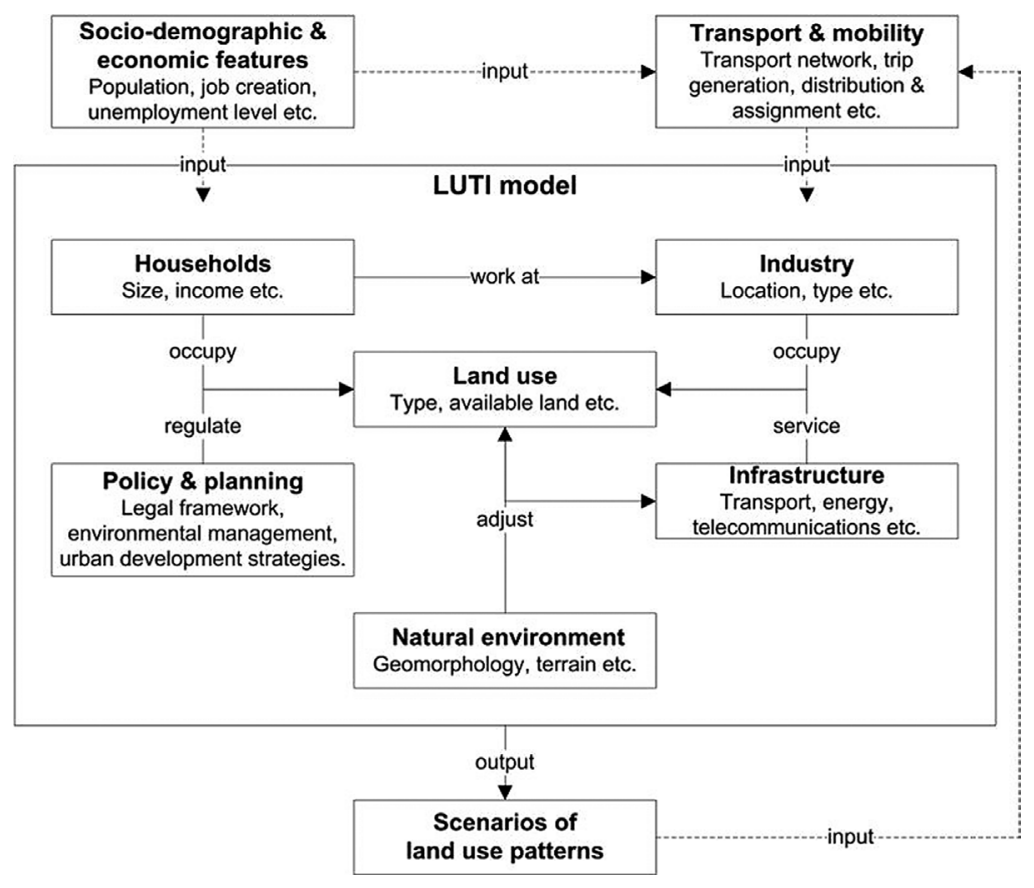

Fig. 1 Structure of a typical LUTI model. 
rate a four stage transportation modeling process i.e. trip generation, trip distribution, modal split and modal assignment and therefore are very suitable for an integrated land use transport approach.

More specifically LUTI models can be used to support strategic planning by estimating trends in locational choices and forecasting land use patterns by combining the features of mobility, socio-demographic characteristics, industry, geomorphology and wider environmental factors, and the availability of urban networks and institutional and policy frameworks (Pozoukidou 2010). According to Fig. 1, the available infrastructure and physical characteristics of the wider urban space and the way these features are taken into account by the planning and policy framework create the conditions that determine where industry is located. These choices affect the locations of households that work in the industry depending on their demographic and socio-economic features and the demands placed on the transport system. In this way, the model is able to produce forecasts of future land use patterns. Thus LUTI models can contribute to strategic transport planning and significally improve the efficiency of the planning process.

Based on this concept various LUTI models with different approaches were developed during the late 1970s and 1980s. Examples are those of Lowry, Putman, Echenique, Anas, Wegener and others (Putman 1992; Wegener 2004; Johnston et al. 2006). However, many of the early models were criticized for being very costly to implement due to the high requirements for collecting and managing data relative to their ability to produce valid and case-specific results. Since then, the development of computers and new technologies that are able produce and manage geo-spatial data through Geographic Information Systems (GIS) and emergence of concepts like sustainability, resilience and holistic planning, are nowadays leading to the enhancement of existing and development of new operational LUTI models.

\section{Description of the LUTI Model Integration Framework}

The proposed framework for the integration of a LUTI model into the SUMP cycle is based on maximising the potential contribution of an integrated land use/transport model for the successful conduction of the aforementioned activities is presented in Fig. 2. There are four (4) phases and eleven (11) Actions that formulate the proposed LUTI integration framework corresponding to the four (4) phases and the appropriate activities of the eleven (11) SUMP steps. More specifically, either the outcome of an Activity of the SUMP cycle (from here on referred to as SUMP Activity) can be used as the input for the corresponding action for the integration of the LUTI model (from here on referred to as LUTI Action) or a LUTI Action can provide outputs for the sup- port of a SUMP Activity, as described in the following sub-sections.

Phase 1-Predictive (Strategy oriented): The first phase of the proposed integration framework aims at the selection and preparation (adjustment) of the appropriate LUTI model and the development of the strategic scenarios. The results from the deployment of strategic scenarios are expected to support the analysis of problems and opportunities, according to the SUMP's first Milestone.

The first LUTI Action is the definition of the model's scope in relation to the needs of the specific study. This action depends on the following SUMP Activities: 1.1 - determining which sustainable mobility principles will be adopted by the plan and how;

1.2 - which involves among others the analysis of the transport and land use policy priorities that need to be taken into account by the model;

1.6 - aims at defining the network of stakeholders from the different transport related sectors.

The next LUTI Action refers to the selection of the most suitable model and its adjustment to the plan's purpose. This depends on the aforementioned scope and on SUMP Activity 1.5, i.e. the setting of the plan's timeline, which will define the dynamic characteristics of the model and the time scale of the short and long-term forecasts.

After the selection of the most suitable LUTI model, the formulation of strategy based scenarios, i.e. a series of scenarios based on the strategic approach of the plan are developed as suggested in SUMP Activity 3.2 (Bührmann et al. 2011). However, in order to formulate resilient and realistic scenarios, one should take into consideration the analysis of problems and opportunities, conducted during SUMP Activity 3.1.

The final action of this phase is the assessment of the strategy based scenarios, which are expected to lead to generic forecasts of urban development patterns according to the urban mobility strategies examined. These forecasts can be exploited in the context of SUMP Activities 4.1 and 5.1, which aim, respectively, to identify the strategic directions and setting of specific priorities for sustainable urban mobility planning. Moreover, the demonstrative capabilities of the model can create a space for discussion among stakeholders and public (SUMP Activity 4.2).

Phase 2-Predictive (Target oriented): During the second phase, the LUTI model can be updated according to the quantified targets set by the second stage of the SUMP in order to provide more detailed forecasts of the possible effects of the selected measures' for the enhancement of urban mobility on the land use system. In this way the model can contribute to the SUMP's second milestone, i.e. the identification of suitable measures.

Specifically, SUMP Activity 5.2 results in the development a series of Specific, Measurable, Achievable, Real- 


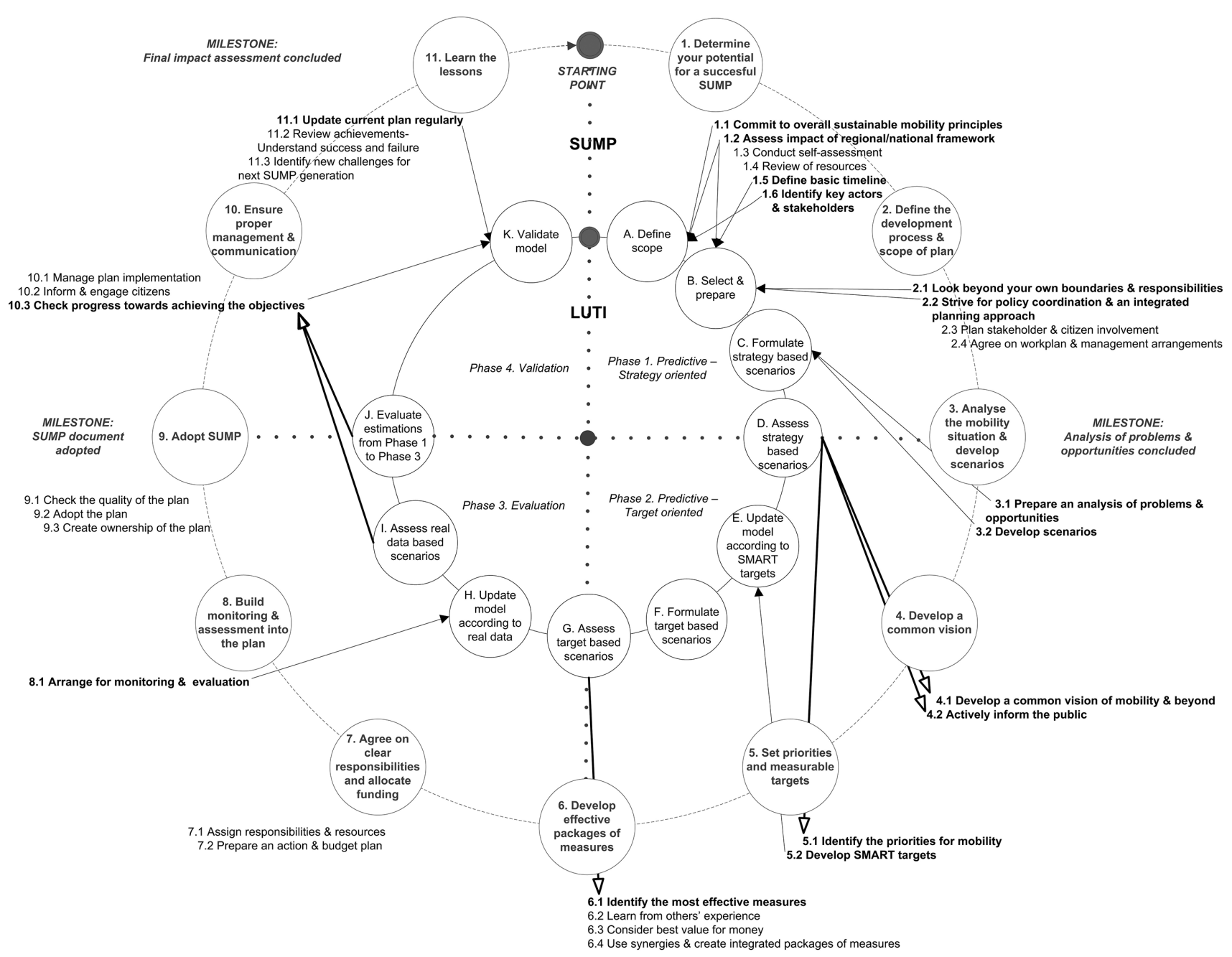

Fig. 2 Framework of the integration of a typical LUTI model into a SUMP cycle.

istic and Time-bound (SMART) targets by selecting and formulating a set of indicators. The corresponding LUTI Action aims at updating the model according to these targets so it is able to produce estimates of indicator values (especially the ones related to the effect of transport on land use) in different time projections. After the formulation of scenarios based on the appropriate combinations of transport related measures and interventions, the target based model can be used to estimate the effect of these measures on the land use system and support the decision making of SUMP Activity 6.1 in identifying the most effective measures.

Phase 3-Evaluation: In this phase the LUTI model is updated based on real data derived from the regular monitoring of indicators during the elaboration of the SUMP and the provision of accurate estimates that can be used to check the progress during the implementation of the SUMP at the milestone in the adoption of the plan.

SUMP Activity 8.1 refers to regular monitoring of a core set of measurable indicators for the evaluation of the plan's elaboration. These measurements can be used as input in the LUTI Action for updating the model.
Then, the updated model can be used to reassess the target based scenarios using real data and reach conclusions on the progress of the implementation of the plan and the achievement of its objectives in terms of the goals related to urban development. Furthermore, the review of the assessment of the use of the model in the strategic, target and real data based scenarios should be made in order to evaluate progress in terms of achieving the objectives of the integrated urban and transport plan.

Phase 4-Validation: The objective of this phase is the overall validation of the contribution of the LUTI model to the SUMP's last milestone, i.e. the assessment of the final effect, and the necessary changes and adjustments for its implementation in the next SUMP. Towards this end, the results and conclusions of the following SUMP Activities,

10.3 - Check progress towards achieving the objectives, 11.1 - Update current plan regularly,

should be embedded in the LUTI Action for the model's validation. This process will ensure that the model is kept up to date with the whole SUMP cycle and is suitable for future use. 


\section{Applications of the Proposed Integration Framework}

It is quite obvious that integration of LUTI models into SUMPS could bring substantial benefits to contemporary strategic planning. Nevertheless application of the proposed framework for the full integration of such models into a SUMP cycle may be limited by several operational bottlenecks.

The most frequent barriers faced during the preparation and implementation of a SUMP is the lack of necessary expertise, absence of political support, limited funds and an inadequate legislative framework. Significant difficulties can also be encountered during the public participation process, a key element in a SUMP. These difficulties mainly concern the limited funding for organizing such processes, the low level of interest and awareness on the part of citizens and stakeholders, the limited tradition in organizing and participating in such processes and the inability of vulnerable groups to express their opinion when up against more influential groups.

Moreover, metropolitan urban areas are in need of a more integrated and comprehensive approach to transport planning. The issues that are related to the transport of persons and goods cannot be addressed by each municipality separately, but requires the existence of a transport authority at the metropolitan level. European legislation provides the framework for the establishment of public transport authorities with broad responsibilities, which include planning, operation and management of multi-modal and alternative transport systems. The advantage of these authorities, which are established in cities like London and Bologna, is that they are staffed with highly qualified multidisciplinary personnel. In contrast, in cities lacking such authorities, overlapping responsibilities and allocation of tasks occur, when at the same time there is lack of appropriate scientific capacity that is particularly needed in the case of the integration of a LUTI model in a SUMP.

The issues associated with the application land use models might be due to reasons that are related to the functionality of the LUTI model per se. These problems are mostly related to the data needed in order for the LUTI models to run the emended calibration and validation processes. The quality of results of these processes are critical for the operation and outcomes of the model and are very dependent on data quality and availability (Pozoukidou 2014). Other concerns about appropriate data are compatibility issues, as the data for LUTI models must be consistent (spatially and temporally) with data used for transport models within a SUMP cycle (Pozoukidou et al. 2015). In addition, there are several usability issues associated with LUTI models, which are extensively discussed in the literature (Vonk et al. 2005). Most of the studies conclude that although these models are commonly used by academics, they are rarely used in policy making and planning. This is due to the fact that these models are conceptually and operationally com- plex and potential users do not have the technical skills or knowledge to use such models (Pozoukidou 2008). To overcome this problem there have been several efforts to develop more user friendly LUTI models that take into account the requirements of policy makers, which can be integrated into the collaborative decision making process.

\section{Conclusions}

This paper demonstrates how LUTI models could be integrated into all four phases of the SUMP process, for the analysis, synthesis and testing of alternative mobility plans. It also demonstrates that integration of a LUTI model into the SUMP cycle is a very efficient means of achieving the strategic goals of the SUMP. Therefore, full integration of LUTI models into a SUMP cycle enhances its strategic and communicative aspects, mainly because LUTI models can be used as testing and evaluating tools, and for communicating and ensuring mutual understanding amongst the stakeholders. Finally, the importance of such integration is related to the fact that assessing the effects of alternative mobility plans on choice of locations, has been recently the core concern of the much desired and theoretically discussed interdisciplinary approach in sustainable transport planning. Nevertheless the success of the proposed approach depends on several operational and institutional aspects that still need to be addressed.

\section{REFERENCES}

Brail R, Klosterman R (2001) Planning Support Systems. Integrating geographical information systems models and visualization tools. ESRI, California.

Bührmann S, Wefering F, Rupprecht S (2011) Guidelines: Developing and implementing a Sustainable Urban Mobility Plan, Eltisplus Project. Intelligent Energy Europe Programme, Rupprecht Consult, Cologne.

EEA. http://www.eea.europa.eu/data-and-maps/indicators/land -take-2. Accessed 16 February 2016.

European Commission (2009) Action Plan on Urban Mobility. European Commission 490, Brussels.

European Commission (2013) A concept for sustainable urban mobility plans. Annex to the Communication from the Commission to the European Parliament, the Council, the European Economic and Social Committee and the Committee of the Regions: "Together towards competitive and resource-efficient urban mobility”. European Commission, Brussels.

Geurs KT, Wee B (2004) Land-use/transport interaction models as tools for sustainability impact assessment of transport. Eur J Transp Infrast 4: 333-355.

Johnston RA, McCoy MC (2006) Assessment of integrated transportation/land use models. Information Center for the Environment, Department of Environmental Science and Policy, University of California Davis.

Pitsiava-Latinopoulou M, Zaharaki E (2004) Transport System and Spatial Development: Theoretical Models and Evaluation. 
In: Kafkalas G (ed.) Issues of Spatial Development. Theoretical Approaches and Policies, Kritiki, Athens, pp. 133-169.

Pozoukidou G, Gavanas N, Verani E (2015) Potential contribution of LUTI models in contemporary strategic planning for urban mobility: a case study of the metropolitan area of Thessaloniki. WIT Transactions on The Built Environment: 168.

Pozoukidou G (2014) Land use transport interaction models: Application perspectives for the city of Thessaloniki. Spatium 32: 7-14.

Pozoukidou G (2010) Land use models: Review and perspective in spatial planning (in Greek). Aeichoros 13:118-140. (in Greek)

Pozoukidou G (2008) Utilisation of urban modeling tools in decision making processes. The TELUM Case Study. In: Psycharis Y, Skayiannis P (eds.) The context, dynamics and planning of urban development: A collection of papers, University of Thessaly Press, Greece, pp. 63-84.

Putman S (1992) Integrated Urban Models 2. London: Pion Limited.
Rodrigue JP (2013) The geography of transport systems. (3rd Edition). Routledge Press, New York.

Seiler A, Folkeson L (2006) Habitat fragmentation due to transportation infrastructure. COST 341 Swedish national state-of-theart report. VTI publishing, Linkoping.

Spiekermann K, Wegener M (2004) Evaluating urban sustainability using land-use transport interaction models. Eur J Transp Infrast 4: 251-272.

U.S. EPA (2000) Projecting Land-Use Change: A Summary of Models for Assessing the Effects of Community Growth and Change on Land-Use Patterns. EPA/600/R-00/098. U.S. Environmental Protection Agency, Office of Research and Development, Cincinnati, $\mathrm{OH}$

Vonk G, Geertman S, Schot P (2005) Bottlenecks blocking widespread usage of planning support systems. Environ Plann A 37: 909-924.

Wegener M (2004) Overview of land-use transport models. Handbook of transport geography and spatial systems 5: 127-146. 